\title{
Mechanism of shock associated with right ventricular infarction
}

\author{
J E Creamer, J D Edwards, P Nightingale
}

\begin{abstract}
Various mechanisms have been proposed to explain the shock sometimes associated with right ventricular infarction, but only small numbers of patients with clinical shock have been studied. The haemodynamic profiles of seven patients with clinical cardiogenic shock after right ventricular myocardial infarction were studied prospectively. They were selected because all had a stable cardiac rhythm and none had absolute hypovolaemia during the study period. In all of them the mean right atrial pressure exceeded the pulmonary artery occlusion pressure. After treatment with varying combinations of dopamine, dobutamine, and glyceryl trinitrate (titrated to achieve the optimum haemodynamic response) the mean systemic arterial pressure increased, as did the cardiac index. There was an associated increase in the left ventricular stroke work index but the right ventricular stroke work index was unchanged. There was no significant change in heart rate, mean right atrial pressure, or pulmonary artery occlusion pressure. This suggests that the probable mechanism of the shock associated with right ventricular infarction is concomitant severe left ventricular dysfunction.
\end{abstract}

Necropsy studies reported in 1948 showed that the right ventricle was often affected in patients with myocardial infarction, ${ }^{12}$ but the clinical syndrome of right ventricular dysfunction associated with inferior myocardial infarction was not fully described until 1974 . $^{3}$ It correlated with extensive involvement of the right ventricle. Subsequently it became apparent that right ventricular infarction may be associated with a range of haemodynamic states. $^{45}$

The frequency of shock associated with right ventricular infarction may be underestimated ${ }^{6}$ but this subgroup of patients with cardiogenic shock has been shown to have a better prognosis and has therefore attracted specific attention. ${ }^{78}$

Several mechanisms of shock in right ventricular infarction have been proposed but their importance has been difficult to assess because this is an unstable condition that requires urgent treatment and does not always allow the rigid application of investigative protocols. Arrhythmias are common in right ventricular infarction and, when present, make an obvious contribution to the development of shock. ${ }^{9}$ Inappropriate administration of diuretic or vasodilator drugs can also precipitate shock. ${ }^{1011}$ Mechanical complications such as rupture of the ventricular septum or free wall and acute mitral regurgitation can produce shock whereas the contributions of tricuspid regurgitation and septal dysfunction are unclear. Hypoxia, by inducing pulmonary hypertension, is probably a significant factor in most patients. ${ }^{8}$ In other patients, though right ventricular dysfunction is regarded as a major cause, particularly in the context of left ventricular filling, ${ }^{11}$ the main debate has been over the contributions of hypovolaemia and concomitant left ventricular dysfunction. ${ }^{12}$

There have been many studies of right ventricular infarction but there are few systematic studies of right ventricular infarction with shock. To define the mechanism of shock associated with right ventricular infarction we examined the haemodynamic profiles of a specific subgroup of patients both before and after successful resuscitation.

\section{Patients and methods}

We prospectively studied seven patients (four men; mean age 62 , range 46 to 74 years) (table) who fulfilled the clinical criteria for cardiogenic shock after transmural inferior myocardial infarction that persisted despite controlled volume replacement where appropriate.

Shock was defined as hypotension (cuff systolic blood pressure $<80 \mathrm{~mm} \mathrm{Hg}$ ) with signs of hypoperfusion (disturbance of conscious level and oliguria with $<20 \mathrm{ml}$ urine per hour), and a cardiac index of $<1.81 / \mathrm{min} / \mathrm{m}^{2}{ }^{13}$ Right ventricular infarction was diagnosed on the basis of electrocardiographic features of acute inferior transmural infarction and associated increases in serum concentrations of creatine kinase and aspartate transaminase, raised jugular venous pressure with paradox, and the absence of clinical and radiological evidence of pulmonary oedema. In all cases invasive monitoring showed raised right atrial pressure that exceeded the pulmonary artery occlusion pressure; there was a steep ' $y$ ' descent in the right atrial pressure waveform and an early diastolic "dip" in the right ventricular pressure trace. ${ }^{13-15}$ During the study period 15 patients with shock and right ventricular infarction, as orginally described, were managed on this unit. The patients reported here were chosen because they had no change in cardiac rhythm between measurements; none had any

South Manchester,

Manchester M20 8LR.

Accepted for publication 2 October 1990 


\begin{tabular}{|c|c|c|c|c|c|c|c|c|c|c|c|}
\hline \multirow[b]{2}{*}{ Casc } & \multirow[b]{2}{*}{ Sex } & \multirow[b]{2}{*}{ Age } & \multirow{2}{*}{$\begin{array}{l}\text { Previous } \\
\text { infarct }\end{array}$} & \multirow{2}{*}{$\begin{array}{l}\text { Other } \\
\text { diseases }\end{array}$} & \multirow[b]{2}{*}{ Drugs } & \multicolumn{2}{|c|}{$\begin{array}{l}\text { Maximum } \\
\text { concentration }(U / l)\end{array}$} & \multirow[b]{2}{*}{$I P P V$} & \multirow[b]{2}{*}{ Arrhythmia } & \multirow{2}{*}{$\begin{array}{l}\text { Inotrope } \\
(\mu g / \mathrm{kg} / \mathrm{min})\end{array}$} & \multirow[b]{2}{*}{ Outcome } \\
\hline & & & & & & $C K$ & $A S T$ & & & & \\
\hline 1 & M & 71 & - & - & 一 & 3257 & 358 & - & CHB & DA $12 \cdot 5$ & $\mathbf{S}$ \\
\hline 2 & $M$ & 48 & - & - & - & 4962 & 500 & + & - & DB 42.7 & $\mathrm{~S}$ \\
\hline 3 & $M$ & 46 & Anterior & CABG & GTN & 3144 & 295 & + & - & DB 13.5 & $\mathrm{~S}$ \\
\hline 4 & $\mathrm{~F}$ & 74 & - & Previous AF & - & 2885 & 319 & + & AF, VT CHB & DA 4.0 & S \\
\hline 5 & $\mathbf{F}$ & 72 & - & Hypertension, angina & $\begin{array}{l}\text { meDOPA, } \\
\text { nifedipine }\end{array}$ & 476 & 221 & + & $\mathrm{CHB}, \mathrm{AF}$ & $\mathrm{DA} \quad 6.0$ & Died \\
\hline 6 & M & 59 & - & $\begin{array}{l}\text { Intermittent } \\
\text { claudication }\end{array}$ & - & 1697 & 264 & + & VF & DA 8.0 & $\mathrm{~S}$ \\
\hline 7 & $\mathbf{F}$ & 62 & Anteroseptal & - & - & 5280 & 850 & + & AF, CHB VF & DA $20 \cdot 2$ & $\mathbf{S}$ \\
\hline
\end{tabular}

$\overline{\mathrm{AF}}$, atrial fibrillation; AST, aspartate aminotransferase; CABG, coronary artery bypass grafting; CHB, 3rd degree atrioventricular block; CK, creatine kinase; DA, dopamine; DB, dobutamine; GTN, glyceryl trinitrate; IPPV, intermittent positive pressure ventilation; Max, maximum; meDOPA, methyldopa; Min, minimum; $\mathrm{S}$, survivor; VF, ventricular fibrillation; VT, ventricular tachycardia.

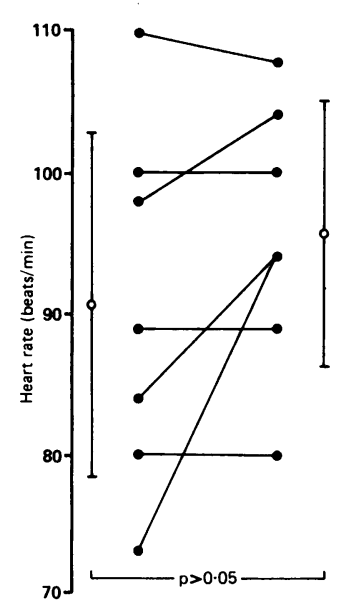

Figure 1 Heart rate (mean (1SD)) before and after reversal of shock.

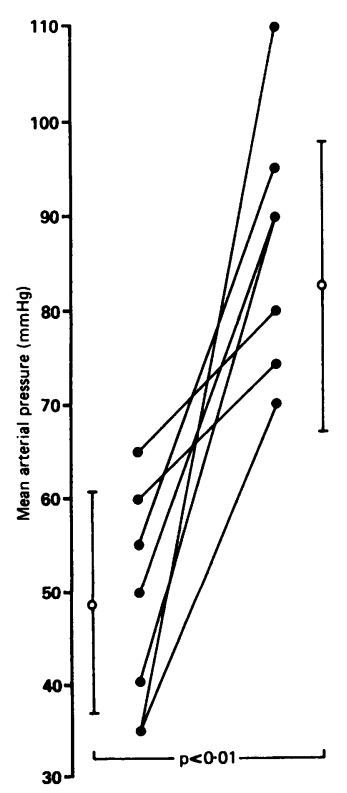

Figure 2 Mean arterial blood pressure (mean (1SD) before and after reversal of shock. evidence of mechanical complications from their infarction or pre-existing respiratory disease. No patient had been treated with diuretics, vasodilators, thrombolytic drugs, $\beta$ adrenergic blocking agents, or other negatively inotropic agents immediately before the onset of shock. There was no change in mode of ventilation during the study.

The time from onset of myocardial infarction to the onset of shock ranged from four to 104 (mean 41) hours.

\section{HAEMODYNAMIC MEASUREMENTS}

All patients had pulmonary artery balloon flotation catheters and femoral arterial catheters inserted by one of us to monitor treatment and to measure intravascular pressures $(7000$ series bedside monitors, Marquette Electronics, Milwaukee, Wisconsin). We measured right atrial pressure, pulmonary artery occlusion pressure, and mean arterial pressure. Cardiac output was measured by a standard thermodilution method with a cooled injectate $\left(<10^{\circ} \mathrm{C}\right)$. Stroke volume index, systemic vascular resistance, left ventricular stroke work index, and right ventricular stroke work index were calculated from standard formulas.

\section{MANAGEMENT PROTOCOL}

The patients were managed according to a protocol that was agreed before the study. Priority was given to the correction of hypoxia (for which six patients needed mechanical ventilation), pacing for complete heart block (four patients), and DC countershock (two patients with ventricular fibrillation and one with ventricular tachycardia). Pulmonary and femoral arterial catheters were inserted as soon as possible and modified fluid gelatin or glyceryl trinitrate was infused, where necessary, to produce an optimum pulmonary artery occlusion pressure of 16 to $18 \mathrm{~mm} \mathrm{Hg} .{ }^{16}{ }^{17}$ For the purpose of this study a full set of haemodynamic measurements was made at this stage and no further fluid load was given. At this point all patients had persistent clinical and haemodynamic features of shock, as defined above, and required additional inotropic support. They were treated with intravenous infusions of either dopamine or dobutamine. Initial doses of dopamine were $2.5 \mu \mathrm{g} / \mathrm{kg} / \mathrm{min}$, and dobutamine $5 \mu \mathrm{g} / \mathrm{kg} / \mathrm{min}$; these were adjusted according to frequent measurements of cardiac output to give a cardiac index approaching $2 \cdot 21 / \mathrm{min} / \mathrm{m}^{2},{ }^{13}$ mixed venous oxygen saturation of $\geqslant 60 \%$, and reversal of the clinical features of shock as previously described. ${ }^{18}$ When these goals had been achieved the haemodynamic measurements were repeated.

Haemodynamic variables before and after oे treatment were compared by Student's $t$ test for paired samples, supplemented by the Wilcoxon signed rank test where the distributions were in doubt (left ventricular stroke work index, systemic vascular resistance).

\section{Results}

Figures 1-4 show the initial haemodynamic measurements and those after reversal of shock. There was no significant change in heart rate when shock was reversed but there were significant increases in mean arterial pressure, stroke volume index, and cardiac index.

There was no significant change in mean systemic vascular resistance, which was 897 (340) dyn.s. $\mathrm{cm}^{-5}$ before treatment and 1052 (186) dyn.s. $\mathrm{cm}^{-5}$ after treatment. Figure 5 shows the change in left and right ventricular stroke work index associated with reversal of shock and its relation to the preload of the respective ventricle. There was no significant change in right atrial pressure $(21$ (3) $\mathrm{mm} \mathrm{Hg}$ before and $23(3) \mathrm{mm} \mathrm{Hg}$ after treatment) or pulmonary artery occlusion pressure (17 (4) $\mathrm{mm} \mathrm{Hg}$ before and 19 (3) $\mathrm{mm} \mathrm{Hg}$ after treatment). Left ventricular stroke work index increased significantly on reversal of shock whereas right ventricular stroke work index was unchanged.

\section{Discussion}

Studies of right ventricular infarction are common whereas those of shock are scarce. ${ }^{1920}$ All the patients we studied had shock after right ventricular infarction, which was diagnosed on the basis of the electrocardiographic changes of inferior infarction and the clinical and haemodynamic signs of right ventricular dysfunction in addition to those of shock. The use of radionuclide or contrast angiographic studies to confirm the diagnosis was not feasible or appropriate in the initial unstable phase of shock during which the studies were performed, but echocardiography was used to ex- 


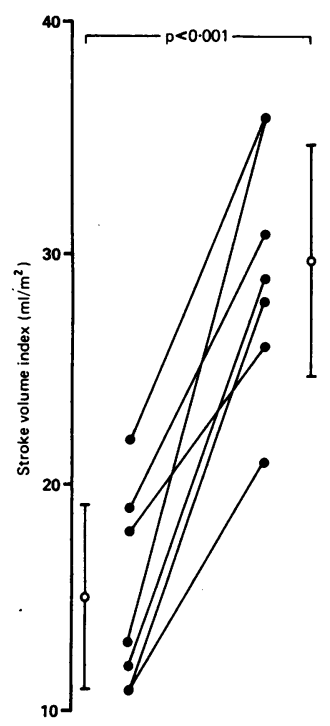

Figure 3 Stroke volume index (mean (1SD)) before and after reversal of shock.

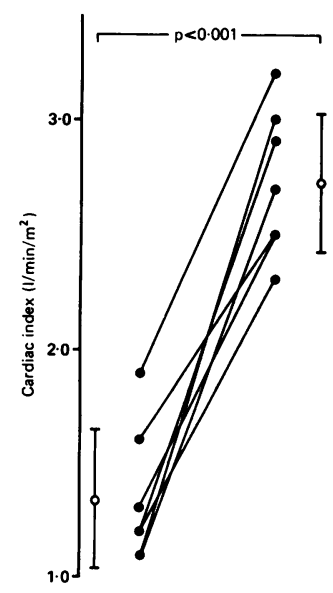

Figure 4 Cardiac index (mean (1 SD)) before and after reversal of shock.

Figure 5 Relation of stroke work index to filling pressure (mean (1 SD)). clude mechanical complications of infarction. Previous reports using such methods for the diagnosis of right ventricular infarction concentrated on less ill patients, though occasional patients with shock were included. ${ }^{21}$ The small number of patients in the present study was unavoidable because of the entry criteria and the difficulty in controlling a study protocol in unstable and critically ill patients. The study was performed before the introduction of routine thrombolytic treatment.

Because all the patients had a stable cardiac rhythm during the study period, the contribution of arrhythmia to the shock state was minimised. Similarly, the potential vasoconstrictive effects of hypoxaemia on the pulmonary vascular bed were minimised by prompt correction of hypoxia, by mechanical ventilation where necessary, before any other treatment. At the time of the study it was not possible to assess specifically the potential influence of acute secondary tricuspid regurgitation or of paradoxical septal motion or right ventricular dilatation in producing shock; but the lack of improvement in right ventricular function when shock was reversed suggests that these factors are unlikely to have contributed significantly.

Hypovolaemia in acute right ventricular infarction can be absolute if there have been excessive fluid losses because of sweating, vomiting, or the inappropriate use of diuretics. ${ }^{810}$ It may also be relative because the optimal left ventricular filling pressure is higher than normal during acute infarction. Allen $e t$ al, relying on central venous pressure monitoring, found relative hypovolaemia in $20 \%$ of patients with cardiogenic shock after myocardial infarction and improved their clinical state by the infusion of up to $300 \mathrm{ml}$ of crystalloid hourly. ${ }^{11}$ Others have sought to improve cardiac performance in acute myocardial infarction by fluid volume expansion controlled by measurement of the left ventricular filling pressure. Russell et al suggested that the optimum left ventricular end diastolic or pulmonary artery disastolic pressure was 20-24 $\mathrm{mm} \mathrm{Hg} .{ }^{22}$ Of the 19 patients studied, however,

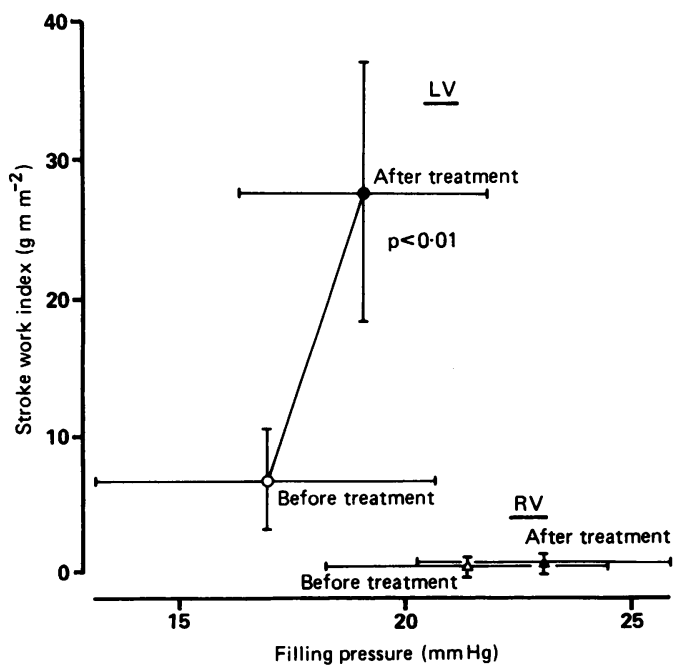

only three were hypotensive. Five patients had no improvement in stroke index with increasing filling pressure, four of these had overt heart failure, and four of this group died. The "optimum" filling pressure was therefore defined for the patients with less severe left ventricular dysfunction but was not clear for this latter group and therefore its application to patients with shock could be considered inappropriate.

Crexells et al measured pulmonary artery occlusion pressure in 23 patients with myocardial infarction (12 with pulmonary congestion and five with cardiogenic shock) and found the optimum to be $14-18 \mathrm{~mm} \mathrm{Hg}{ }^{17}$ The cardiac index and stroke work index could be improved by fluid volume expansion only when the initial pressure was $<14 \mathrm{~mm} \mathrm{Hg}$. They also found that right ventricular function was improved by increased right atrial pressure but only for increases of up to $6-8 \mathrm{~mm} \mathrm{Hg}$. The anatomical site of infarction was not specified in this study. A recent study of volume loading and glyceryl trinitrate administration in right ventricular infarction suggested that the optimal filling pressure of the right ventricle was 10 to 14 $\mathrm{mm} \mathrm{Hg} .{ }^{23}$ Above this value right ventricular stroke work index declined. In patients with shock after right ventricular infarction the right ventricular filling pressure is almost invariably higher than this.

In occasional patients with right ventricular infarction and shock, fluid volume expansion alone restored normal systemic perfusion. This is a rare effect in patients who have not previously received diuretics. ${ }^{8}$ Because of the design of the present study achieving an optimum left ventricular filling pressure alone was insufficient to reverse the haemodynamic and clinical features of shock, and in all cases additional inotropic support was required.

The role of right ventricular dysfunction in the production of shock was suggested in early reports of right ventricular infarction. This was based on the assumption that any associated left ventricular dysfunction was modest because pulmonary artery occlusion pressures were low and the condition apparently responded well to administration of fluid. It has been suggested that the reduced pump function of the right ventricle may impair left ventricular filling. ${ }^{24} 25$ A study of prognosis of postinfarction ventricular septal rupture found a higher mortality in patients with inferior infarctions. ${ }^{26}$ Shock was more common in patients with inferior infarctions and it correlated with mortality; from this it was suggested that right ventricular dysfunction was responsible. Indirect methods were used to assess the function of each ventricle, however, and the role of right ventricular dysfunction in the production of shock was not established.

In right ventricular infarction, if pulmonary arterial hypertension is not present because of hypoxia, there is a progressive pressure gradient from right atrium through right ventricle and pulmonary arteries to pulmonary veins and left atrium that allows passive blood flow. ${ }^{12} 27$ Furthermore, the pressure waveforms in right atrium, ventricle, and pulmonary 
artery are often indistinguishable in acute right ventricular infarction (which may require fluoroscopy to position the pulmonary arterial catheter) suggesting that passive flow may indeed occur. In the present study the reversal of clinical shock with improvement in cardiac output and with increases in left ventricular stroke work index strongly suggest that reversal of shock was brought about by improvement in left ventricular function and that the shock was primarily related to this dysfunction.

In this clinical study of patients in cardiogenic shock we were unable to study the causes of this left ventricular dysfunction but we can postulate possible mechanisms. Firstly, as originally shown in necropsy studies, isolated right ventricular infarction occurs in only 2$4 \%$ of cases. ${ }^{12}$ There is almost always concomitant disease of the left coronary artery with varying degrees of left ventricular infarction. This leads to loss of contracting myocardium and to a reduction in left ventricular compliance. In response to shock the systemic vasculature responds by vasoconstriction. This increased afterload might further lower stroke volume. In the presence of a grossly raised right atrial pressure caused by right ventricular infarction the formula for calculation of systemic vascular resistance may underestimate the true level of peripheral vascular tone. Right ventricular dilatation may further reduce left ventricular compliance through the constraining influence of the pericardium. This may be worsened by overenthusiastic and possibly ill advised use of fluid infusion. Hypoxia may extend the degree of ischaemic damage to the left ventricle and is a frequent finding in shock caused by right ventricular infarction, even in the absence of pulmonary oedema. ${ }^{8}$ Hypoxia will increase peripheral vascular tone and further depress left ventricular function by increasing the afterload.

The improvements in left ventricular performance that were brought about by the use of inotropes in the current study may have been because conditions for their use were optimised by correction of hypoxia, rational use of volume loading or nitrates to produce an optimal preload, and careful titration of the dosage guided by constant clinical and haemodynamic evaluation.

In the present study, reversal of shock was achieved without any increase in right ventricular stroke work index. This suggests that right ventricular dysfunction was not primarily responsible for the state of shock.

Animal experiments showed that complete ablation of the right ventricle had little demonstrable effect on resting venous or arterial pressure; however, volume loading was not used in the early studies. ${ }^{2829}$ These experiments usually involved removal of the restraining influence of the pericardium.

Without additional cardiovascular stress extensive replacement of right ventricular myocardium by fibrous and adipose tissue in the rare Uhl's anomaly can be consistent with adequate haemodynamic function, and it has been suggested that the pulmonary blood flow can be maintained by right atrial contraction. ${ }^{30}$

Some support for our hypothesis comes from a close examination of the results of a previous study of patients in shock. Gewirtz et al compared the initial haemodynamic profiles of patients with inferior myocardial infarction who had cardiogenic shock with those without shock. ${ }^{31}$ The shock patients were divided into those with and those without right ventricular dysfunction. Though right ventricular stroke work index was reduced in those with right ventricular dysfunction, the presence of shock was associated with a considerable reduction in left ventricular stroke work index in both groups. This showed that left ventricular dysfunction may be more extensive than had been thought. Plasma volume expansion to produce an optimum pulmonary artery occlusion pressure did not improve the overall cardiac index in three of the six patients with shock and righ ventricular dysfunction, all of whom died. Three patients survived but two required intra-aortic balloon counterpulsation. The role of reversal of left ventricular dysfunction was not formally documented in Gewirtz et al's study.

In the current study we confirmed the considerable reduction of left as well as right ventricular stroke work indices, but we also showed that reversal of shock was accompanied by an increase in left ventricular stroke work index while right ventricular stroke work index remained unchanged, which suggests that the left ventricular dysfunction was responsible for the shock. In practice, the need to achieve and maintain an optimal left ventricular filling pressure should not be underestimated, but in those patients whose shock is not due to diuretics or vasodilators volume expansion should be controlled for the reasons described above.

1 Wartman WB, Hellerstein HK. The incidence of hear disease in 2,000 consecutive autopsies. Ann Intern Med 1948;28:41-65.

2 Yater WM, Traum AH, Springs S, et al. Coronary artery disease in men 18-39 years of age. Am Heart $J$ 1948;50 683-722.

3 Cohn JN, Gulha NH, Broder MI, Constantinos JL. Righ ventricular infarction-clinical and hemodynamic features. Am J Cardiol 1974;33:209-14.

4 Cintron GB, Hernandez E, Linares E, Aranda JM. Bedside recognition, incidence and clinical course of right venrecognition, incidence and clinical course of right ven-
tricular infarction. Am J Cardiol 1981;47:224-7.

5 Rackley CE, Russell RO, Mantle JA, Rogers WJ, Papapietro SE, Schwartz RM. Right ventricular infarction and SE, Schwartz RM. Right ventricular

6 Scheidt S, Ascheim R, Killip T. Shock after myocardia infarction, a clinical and hemodynamic profile. $\mathrm{Am}$ Cardiol 1970;26:556-64

7 Lloyd EA, Gersh BJ, Kenelly BM. Hemodynamic spectrum of "dominant" right ventricular infarction in 19 patients. Am J Cardiol 1981;48:1016-22.

8 Edwards D, Whittaker S, Prior A. Cardiogenic shock without a critically raised left ventricular end diastolic pressure: management and outcome in eighteen patients. Br Heart $J$ 1986;55:549-53.

9 Love JC, Haffajee CI, Gore JM, Alper JS. Reversibility of hypotension by atrial or atrioventricular sequential pacin in patients with right ventricular infarction. Am Heart 1984;108:5-13.

10 Come P, Pitt B. Nitroglycerin-induced severe hypotension and bradycardia in patients with acute myocardial infarction. Circulation 1976;54:624-8.

11 Allen HN, Danzig R, Swan HJC. Incidence and significance of relative hypovolemia as a cause of shock associated with acute myocardial infarction [Abstract]. Circulation 1967; 36(suppl II):50.

12 Cohn JN. Right ventricular infarction revisited. $\mathrm{Am}$ 
Cardiol 1979;43:666-8.

13 Forrester JS, Diamond G, Chatteriee K, Swan HJC Medical therapy of acute myocardial infarction by application of hemodynamic subsets. $N$ Engl J Med 1976;295: $1356-62$ and $1404-13$

14 Lorell B, Leinbach RC, Pohost GM, et al. Right ventricular infarction-clinical diagnosis and differentiation from cardiac tamponade and pericardial constriction. Am J cardiac tamponade and
Cardiol 1979;43:465-71.

15 Isner JM. Right ventricular myocardial infarction. JAMA 1988;259:712-8.

16 Shoemaker WC. Relation of oxygen transport patterns to the pathophysiology and therapy of shock states. Intensive Care Med 1987;13:230-43.

17 Crexells C, Chatterjee K, Forrester JS, Dikshit K, Swan HJC. Optimal level of filling pressure in the left side of the heart in acute myocardial infarction. N Engl J Med 1973, 24:1263-6.

18 Creamer JE, Edwards JD, Nightingale P. Hemodynamic and oxygen transport variable in cardiogenic shock secondary to acute myocardial infarction and its response to treatment. Am J Cardiol 1990;65:1297-300.

19 Sharpe DN, Botvinick EH, Shames DM, et al. The non-invasive diagnosis of right ventricular infarction non-invasive diagnosis of
Circulation 1978;57:483-90.

20 Tobinick E, Schelbert HR, Henning H, et al. Right ventricular ejection fraction in patients with acute anterio and inferior myocardial infarction assessed by radionuclide angiography. Circulation 1978;57:1078-84.

21 Rigo P, Murray M, Taylor DR, et al. Right ventricular dysfunction detected by gated scintigraphy in patients with acute inferior myocardial infarction. Circulation 1975;52:268-74.

22 Russell RO, Rackley CE, Pombo J, Hunt D, Potanin C Dodge HT. Effects of increasing left ventricular filling pressure in patients with acute myocardial infarction. $J$ Clin Invest 1970;49:1539-50.

23 Berisha S, Kastrati A, Goda A, Popa Y. Optimal value of filling pressure in the right side of the heart in acute right ventricular infarction. Br Heart J 1990;63:98-102.

24 Dell'Italia LJ, Starling MR, Crawford MH, et al. Right ventricular infarction: identification by hemodynamic measurements before and after volume loading and measurements before and after volume loading and
correlation with non-invasive techniques. J Am Coll correlation with non-in
Cardiol 1984;4:931-9.

25 Goldstein JA, Vlahakes GJ, Verreir ED, et al. Volume loading improves low cardiac output in experimental right ventricular infarction. J Am Coll Cardiol 1983;2:270-8.

26 Moore CA, Nygaard TW, Kaiser DL, Cooper AA, Gibson RS. Post-infarction ventricular septal rupture: the importance of location of infarction and right ventricular function in determining survival. Circulation 1986;74: 45-55.

27 Kulbertus HE, Rigo P, Legrand V. Right ventricular infarction: pathophysiology, diagnosis, clinical course and treatment. Mod Concepts Cardiovasc Dis 1985;4:1-5.

28 Starr I, Jeffers WA, Meade RH. The absence of conspicuous increments of ventricular pressure after severe damage to the right ventricle of the dog, with a discussion of the relation between clinical congestive failure and heart relation between clinical congestive
disease. Am Heart $J$ 1943;26:291-301.

29 Guiha N, Limas CJ, Broder MI, Cohn JN. Predominant right ventricular failure in clinical and experimental right ventricular infarction [Abstract]. Clin Res 1972;20:375.

30 Vecht RJ, Carmichael DJS, Gopal R, Philip G. Uhl's anomaly. Br Heart J 1979;41:676-82.

31 Gewirtz H, Gold HK, Fallon JT, Pasternak RC, Leinbach $\mathrm{RC}$. Role of right ventricular infarction in cardiogenic shock associated with inferior myocardial infarction. $\mathrm{Br}$ Heart $J$ 1979;42:719-25. 